\title{
Spinning and Processing Continuous Yarns from 4-Inch Wafer Scale Super-Aligned Carbon Nanotube Arrays**
}

\author{
By Xiaobo Zhang, Kaili Jiang, * Chen Feng, Peng Liu, Lina Zhang, Jing Kong, Taihua Zhang, \\ Qunqing Li, and Shoushan Fan*
}

Carbon nanotubes (CNTs) are an extremely interesting type of material due to their unique 1D structure, and their excellent mechanical, thermal, and electrical properties. ${ }^{[1]}$ Many promising applications have been demonstrated. ${ }^{[2]}$ To exploit their excellent physical properties at a macroscopic level, it is desirable to create CNTs with macroscopic length. However, it has been very challenging to grow arbitrarily long CNTs. ${ }^{[3]}$ An alternative approach is to create long nanotube structures with many of them aligned into continuous yarns or ropes. $^{[4-7]}$

In 2002, a breakthrough was made by our group to fabricate pure CNT yarns by directly drawing CNTs from super-aligned CNT arrays. ${ }^{[5]}$ Following that, Zhang et al. developed a drawtwisting spin method ${ }^{[6]}$ and demonstrated more interesting applications of the raw yarns. ${ }^{[7]}$ Since CNTs in the yarn are nearly parallel aligned, the CNT yarn is intrinsically an anisotropic material and has a special axis along the drawing direction, which demonstrates many fascinating properties and applications such as filaments for light bulbs, ${ }^{[5]}$ polarizers working in the UV region, ${ }^{[5]}$ thermal-field emitters, ${ }^{[8]}$ polarized-light emitters, ${ }^{[7]}$ transparent conducting membranes, ${ }^{[7]}$ etc. As these yarns are macroscopic objects, there is no doubt that they will be sought after for more and more applications as time goes on.

[*] Prof. K. Jiang, Prof. S. Fan, X. Zhang, C. Feng, P. Liu, Dr. L. Zhang, Prof. Q. Li

Department of Physics

and Tsinghua-Foxconn Nanotechnology Research Centre

Tsinghua University

Beijing 100084 (P.R. China)

E-mail: jiangkl@tsinghua.edu.cn; fss-dmp@tsinghua.edu.cn

Prof. J. Kong

Department of Electrical Engineering and Computer Science

Massachusetts Institute of Technology

Cambridge, MA 02139-4307 (USA)

Prof. T. Zhang

LNM, Institute of Mechanics

Chinese Academy of Sciences

Beijing 100080 (P.R. China)

[**] K. Jiang, Q. Li, and S. Fan acknowledge financial support from the National Basic Research Program of China (2005CB623606) and the NSFC (10334060). J. Kong acknowledges financial support from the MARCO program by the interconnect focus center. T. Zhang acknowledges financial support from the NSFC (10572142, 10372103). We thank Tengge Ma and Liguo Xu for help with the e-beam evaporation of the iron catalyst, Jiahe Tian for help with the LP-CVD system, and Manqiong Xu for help with the SEM experiments. Supporting Information is available online from Wiley InterScience or from the author.
However, to achieve real applications in the industry, some key issues have to be solved in advance. Currently many groups have achieved the growth of CNT arrays, ${ }^{[9,10]}$ but of these only two groups have reported spinning yarns from their arrays. $^{[5-7]}$ The first question is why our CNT arrays can give rise to CNT yarn while others cannot? What is the critical factor that determines the ability for yarn formation? In our first paper $^{[5]}$ and the recent paper of Zhang et al., ${ }^{[6,7]}$ the reported syntheses were carried out at atmospheric pressure (AP) in a tube furnace with diameters of 1-2 in. Can this synthesis be expanded to a larger scale at low pressure (LP) for commercial chemical vapor deposition (CVD) systems? The third problem is that, even though many applications have been demonstrated, the directly drawn-out yarns are very sticky due to their clean surfaces and extremely high surfaceto-volume ratio. It can easily to stick to the surfaces of other objects and can never be separated again, which greatly inhibits the real application of CNT yarns. Here, we show how these crucial problems were tackled by our group in the past two years.

CNT arrays (or termed "CNT forests") represent high-quality and highly ordered CNT structures, in which CNTs are nearly parallel aligned and perpendicular to the substrate. To date, a lot of groups have successfully synthesized CNT arrays since the initial papers were published. ${ }^{[9,10]}$ However, only two groups have reported that CNT yarns can be drawn from their arrays, ${ }^{[5-7]}$ which were defined as super-aligned arrays by us. ${ }^{[5]}$ We have tried to pull yarns from CNT arrays derived by a previously published method, ${ }^{[10]}$ which is denoted as a normal array, no yarns could be drawn out even though the height of the CNT array was above $1 \mathrm{~mm}$. Now the question is what the difference is between normal and super-aligned arrays?

Zhang et al. claimed that the formation of yarn was due to the disordered region at the top and bottom of the CNT array, which entangled together forming a loop. ${ }^{[6,7]}$ However we cannot agree with them based on the following points. First, in the normal array, the top and bottom part are more disordered and entangled than in the super-aligned array. Second, in our super-aligned array, the bottom part is highly ordered (see supporting information, Fig. S1) and without entanglement. Therefore, the disordered entanglement at the top may help the formation of yarn, but it cannot be the key factor for yarn formation.

To elucidate the difference between normal and superaligned arrays, we performed comparative studies. Figure 1A is the transmission electron microscopy (TEM) image of 

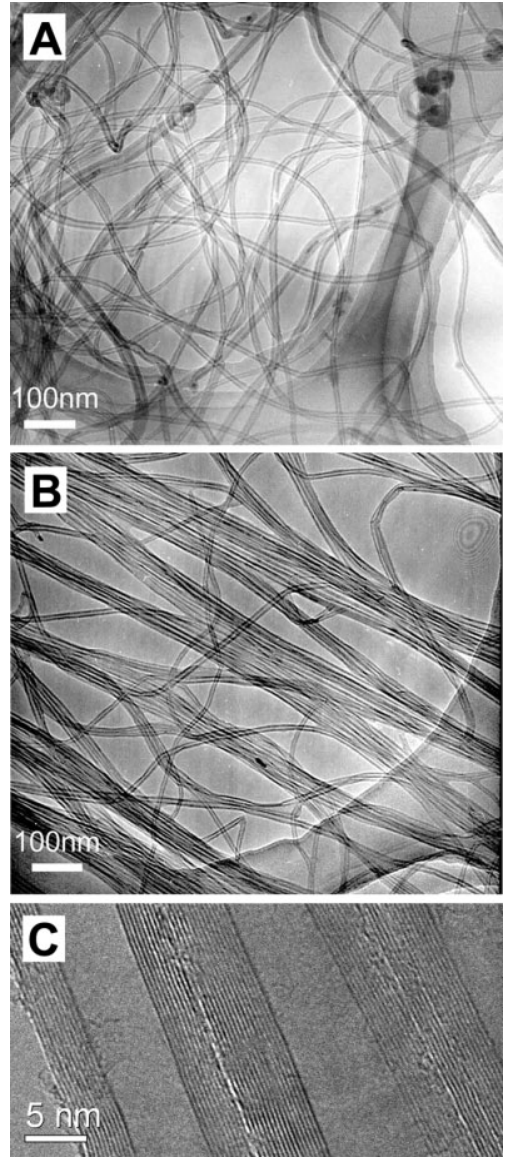

Figure 1. TEM images of CNTs in A) normal arrays, B) super-aligned arrays. C) HRTEM image of as-grown CNTs from super-aligned arrays.

CNTs derived from the normal arrays, which are synthesized by using a previously reported method. ${ }^{[10]}$ Figure $1 \mathrm{~B}$ shows the TEM image of CNTs derived from super-aligned arrays. It is clear that CNTs from normal arrays are isolated from each other while CNTs from super-aligned arrays form tight bundles, indicating strong van der Waals interactions between the tubes. We therefore believe that CNTs in super-aligned CNT arrays have very clean surfaces, which is confirmed by the high-resolution TEM (HRTEM) of as-grown CNTs from the super-aligned arrays (Fig. 1C). Therefore, the unique features of super-aligned CNT arrays are that the CNTs have very clean surfaces, and consequently there are strong van der Waals interactions between them. When pulling the CNTs from a super-aligned array, it is the van der Waals force that makes the CNTs join end to end, thus, forming a continuous yarn, which is illustrated in Figure 2E.

To further validate our ideas, we recorded the pulling yarn process under a scanning electron microscope (see supporting information, Fig. S2) and an optical microscope (see supporting information, Fig. S3). From these observations it can be seen that CNT bundles are peeled off from the super-aligned array, indicating a strong binding force between the bundles and the array. Also the bundles were joined end to end forming a continuous yarn. It is clear that the model we proposed (Fig. 2E) basically reflects the true situation of an individual thread in the yarn. There are also some CNT bundles bridging these threads, forming a continuous ribbon several micrometers thick and several centimeters wide. The bridging effect dramatically enhanced the robustness of the yarn. Also the junction part shown in Figure 2E can be clearly identified in scanning electron microscopy (SEM) images of the yarn, as shown in Figure 2G.

To achieve real applications of CNT yarns in industry, it is critical that the synthesis of super-aligned CNT arrays can be scaled up. The major problem in scaling up is the question of how to guarantee the uniformity of the as-grown arrays. To solve this problem, it is highly recommended to achieve the synthesis in commercial LP-CVD, which gives rise to a higher degree of uniformity. In our first paper ${ }^{[5]}$ and in a recent paper of Zhang et al., ${ }^{[6,7]}$ the reported syntheses were carried out at atmospheric pressure (AP) in a tube furnace with diameters of 1-2 in. In the past two years, we have focussed on the scale-up of a synthesis procedure in a LP-CVD system. Recently, we successfully achieved a 4-in. wafer scale synthesis of super-aligned CNT arrays (Fig. 2A) in a LP-CVD system. Detailed considerations on the experimental parameters can be found in the Experimental section.

In 2002 we demonstrated that CNT yarns can be manually drawn from the super-aligned arrays with tweezers. ${ }^{[5]}$ We have developed a more controllable method, i.e., using an electrical motor to perform the pulling with a constant speed and direction. Figure 2B shows that continuous CNT yarn can be pulled from the 4-in. super-aligned array using an electric motor.

The freshly drawn yarns, which appear as very thin ribbons typically several micrometers thick and several centimeters wide, are very sticky due to their clean surfaces and extremely high surface-to-volume ratio. Once contacting with the surface of another object, it will stick to this surface and can not be taken off again. This problem greatly inhibits real applications of the CNT yarn.

To solve this problem, a new method was invented to process the yarns. After being drawn out from the super-aligned array, as is shown in Figure 2B, the CNT yarn was subsequently pulled through droplets of ethanol, after which the several centimeters wide yarn shrank into a tight fiber typically $20-30 \mu \mathrm{m}$ in diameter (Fig. $2 \mathrm{~F}, \mathrm{H}$, and I). Due to its reduced surface area, the treated yarn is no longer as sticky as the fresh yarn, and can be easily wound on and off a spool using a motor or by hand (Fig. 2B). Also the strength of the CNT yarn was dramatically improved, which will be shown later.

The mechanism of this process is not very clear right now. We suspect that surface tension plays a vital role in this process. To our knowledge, CNTs are hydrophobic but can be wetted by most of volatile organic solvents, such as methanol, ethanol, acetone, dichloroethane, chloroform, etc. We have tested all these solvents in the aforementioned process, and have found that all can be employed to process the raw yarn. One possible explanation is that the meniscus around the yarn pinched the yarn tightly when the yarn was being pulled out from the solvent, as is shown in Figure $2 \mathrm{C}$ and $\mathrm{D}$ and the video (S4) in the Supporting Information. 


\section{ADVANCEP \\ MATERIALS}
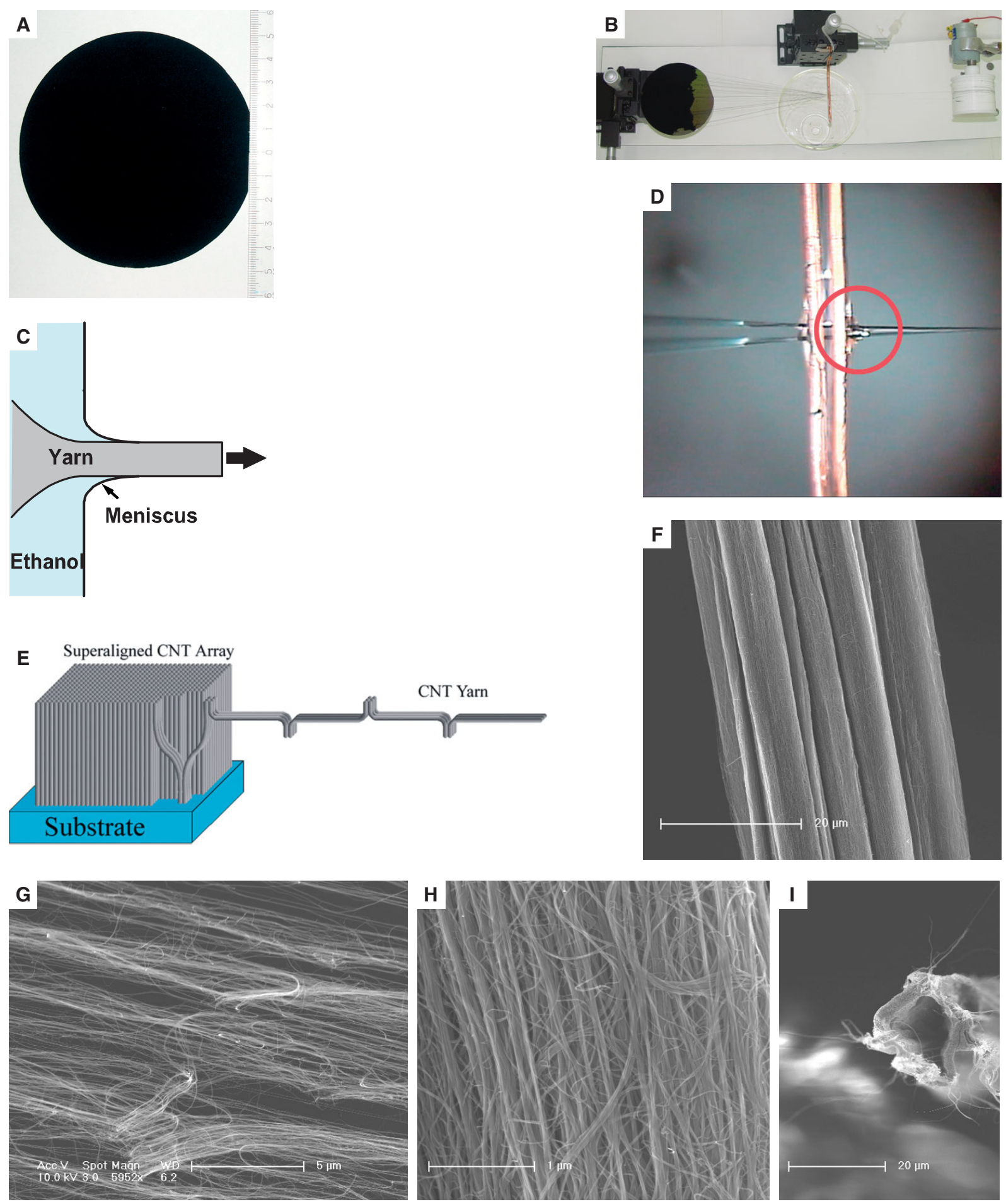

Figure 2. Spinning continuous yarns from super-aligned CNT arrays. A) As-grown super-aligned CNT arrays on 4 inch wafer. B) Spinning continuous yarns from super-aligned CNT arrays. C) Schematic illustration of processing yarn in ethanol, showing the pinch effect of the meniscus. D) Two yarns merging into one tight fiber after passing through an ethanol droplet. E) Pulling yarn model. F) SEM image of yarn after passing through ethanol. Scale bar $20 \mu \mathrm{m}$. G) SEM image of freshly drawn yarns. Scale bar $5 \mu \mathrm{m}$. H) A higher magnification SEM image of (F), scale bar $1 \mu \mathrm{m}$. I) The cross section of CNT yarns treated by ethanol, scale bar $20 \mu \mathrm{m}$.

Note that the volatile solvent is easily evaporated, leaving the pure CNT yarn free of contamination after processing. There are several advantages to this processing method: First and most importantly, the processed yarn is no longer sticky due to a reduced surface area and it can be easily manipulated by hand, which makes it much more convenient to freely con- 
struct macroscopic objects with any desired shape. As a demonstration, a processed yarn was bent to an angle and mounted on two electrodes serving as a thermal electron emitter, which can be easily installed to replace a tungsten wire hot cathode working at above $2000{ }^{\circ} \mathrm{C} .^{[8]}$

The second advantage is that the strength of processed CNT yarns was dramatically improved. Figure 3 shows the tensile testing results of a CNT yarn after ethanol treatment and after further heat treatment. The ethanol-processed yarn

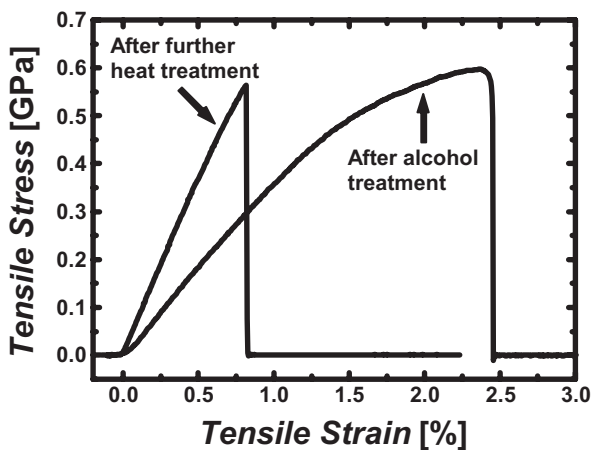

Figure 3. Mechanical properties of CNT yarns. Stress-strain curves of ethanol-treated and further heat-treated CNT yarns tested using an Instron 5848 microtester at a strain rate of $2 \% \mathrm{~min}^{-1}$ for a three-centimeter gauge length.

fails at a strain of $2.45 \%$. The tensile strength is approximately $600 \mathrm{MPa}$ (see Experimental section). This result is higher than that of the single and double poly-yarns reported by Zhang et al., ${ }^{[6]}$ and slightly lower than that of poly(vinyl alcohol) (PVA) infiltrated multi-walled nanotube (MWNT) yarns. ${ }^{[6]}$ We further heated the yarn to about $2000 \mathrm{~K}$ for several hours by electric current, after which the yarn became stiffer than before. The tensile testing result is shown in Figure 3. The tensile strength for the heat-treated yarn is $564 \mathrm{MPa}$, which is slightly smaller than the yarn without heat treatment. This decreasing in tensile strength may originate from the current heating method. As we know, if there is a defect in the yarn, an extraordinary large amount of joule heat will be released here, which will subsequently enlarge the defect via evaporation of carbon atoms. This will eventually break the yarn as time goes on. The current heat-treatment method was chosen because we lacked a high-temperature vacuum furnace. However, the Young's modulus is dramatically enhanced after heat treatment (74 GPa), and is twice as high as that of the untreated yarn. We attribute this enhancement to the welding effect at the joint part shown in Figure 2E, because the strain-to-failure is greatly decreased while the Young's modulus is increased.

Lastly, this processing method can also be employed to combine two yarns together forming one thicker yarn, which is shown in Figure 2D. Furthermore, by simply passing through the solvent droplet simultaneously, two or more processed yarns can be merged together forming a thicker one.
One of the unique features of these processed yarns is that they are both elastic and remarkably pliable. As will be shown below, the processed yarn can be easily wound and manipulated without any damage, compared to carbon fibers, which are very fragile and break easily under bending. The difference between the yarns and carbon fibers is very similar to the difference between a steel wire-rope and a steel rod. The small diameter and weak intertube bonding make it easier to eliminate the bending stress via repositioning of the CNTs. In addition, the nanotubes in our CNT yarns are easily buckled under bending stress due to their tube structure, which is also very helpful for eliminating the bending stress.

Another unique feature is the memory effect after heat treatment, i.e., if we mould the CNT yarn to a certain shape, the shape will be remembered after the heat treatment. This memory effect is probably due to the welding effect at the joint part as mentioned before, which will fix the shape after heat treatment.

These excellent properties mentioned above enabled us to manipulate the yarns easily and make any desired shape. As a demonstration, we constructed a spring by using processed CNT yarns. A more than $3 \mathrm{~m}$ long yarn was first cut into 10 segments with equal length. Then these segments were aligned parallel to each other and passed through an ethanol droplet. As a result, the 10 segments merged into one thick rope, which was subsequently wound on a sapphire rod with a diameter of $5 \mathrm{~mm}$ (Fig. 4A). If the two ends of the rope are not fixed, the rope is automatically unwound again due to the elasticity. We therefore fixed the two ends on two copper electrodes by silver paste, as is shown in Figure 4A. Then a DC current was applied to heat the rope to about $2000 \mathrm{~K}$ (see Fig. 4B) in vacuum for several hours.

When the rope was taken off the sapphire rod, it retained its helical shape, forming a free-standing CNT spring (Fig. 4C). This CNT spring, which is $5 \mathrm{~mm}$ in diameter and several centimeters in length, can be easily and elastically stretched to twice its length. When the external force disappears, the CNT spring will be restored to its original length. Figure 4D shows three repeating results of the tensile testing for the CNT spring, indicating its good elasticity and the repeatability of the process. Note that the noise superposed on the tensile curve originates from the electronic circuits of the testing machine. The three results fall within the noise level of the machine. According to the testing results the CNT spring, which weighs $1.6 \mathrm{mg}$, has a spring constant of $0.1 \mathrm{~N} \mathrm{~m}^{-1}$. This small spring constant is of the same order as that of AFM tips. Therefore, this CNT spring can serve as a force sensor to weigh tiny objects. In fact, this memory effect can be conveniently employed to construct various macroscopic objects with any desired shape.

In summary, the key factor that determines the CNT arrays' ability for yarn formation was identified to be able to keep the surfaces of the CNTs clean. By following this principle, we have successfully scaled up the production of super-aligned CNT arrays on 4 in. silicon wafers, and extended the synthesis from AP to LP-CVD, which is compatible with current semi- 

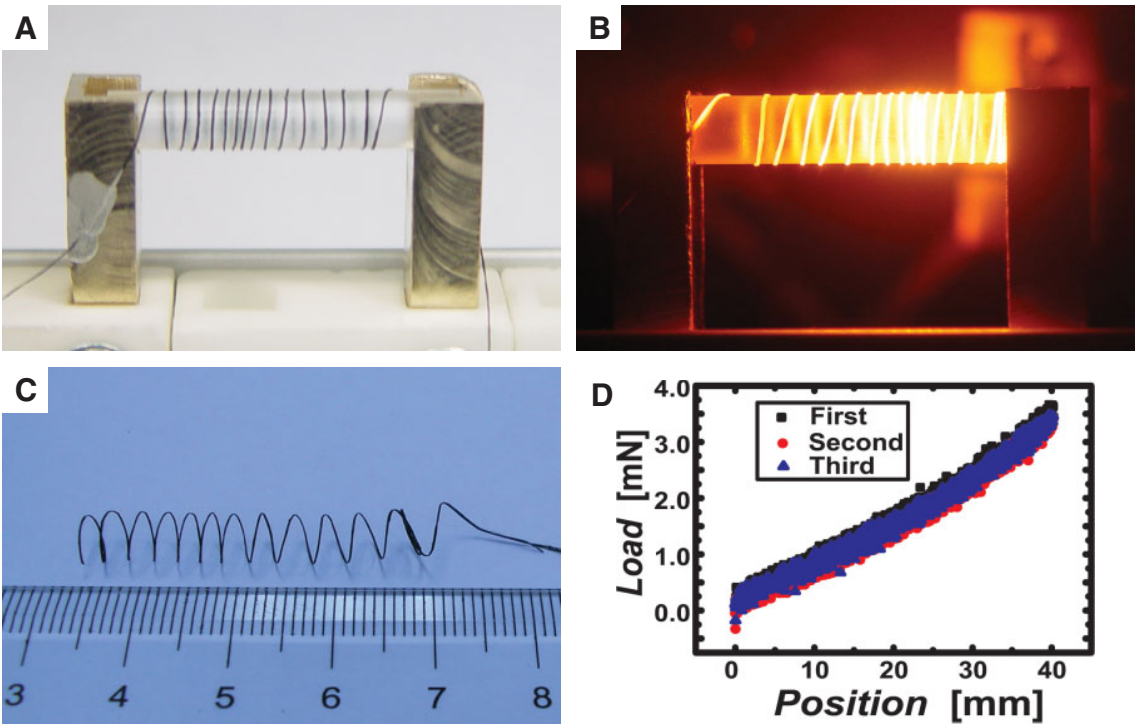

Figure 4. Making a CNT spring by using CNT yarns. A) CNT yarns wound on a sapphire rod. Two ends are fixed on the copper electrodes by silver paste. B) Heat treatment of CNT yarns by an electric current. C) CNT yarns turned into the CNT spring after heat treatment. D) The results of three repeated tensile tests, showing good repeatability and elasticity.

conductor processes. A novel method was used to process the as-drawn CNT yarns, by which the CNT yarns could be turned into a tight thread showing excellent mechanical properties. The processed yarn is both elastic and pliable and can be freely manipulated and molded to any desired shape, which will be retained after heat treatment. Owing to the highly ordered macroscopic structures of 1D nanomaterials, good electrical and thermal conductivity of the nanotube components, good mechanical properties, and extremely facile manipulability, the processed yarn will have much more versatile applications in the future.

\section{Experimental}

Since the key factor for yarn formation is the strong van der Waals interaction between the CNTs, the principle for controlled synthesis of super-aligned CNT arrays is to keep the surfaces of the CNTs clean during growth. As is well known, for CNT growth by a CVD method, hydrocarbon precursors are first cracked by a catalyst heterogeneously and then carbon atoms are incorporated into the CNTs after transportation through the catalyst. This is the normal axial growth process of CNTs. However, there are other processes involved in the CVD growth of CNTs. The first one is that hydrocarbon molecules decompose over the surface of the CNTs, another one is that pyrolysed carbon atoms can directly be deposited on the surface. Both processes result in the formation of amorphous carbon (AC) over newly grown surfaces, which will substantially decrease the van der Waals force between the CNTs. Therefore, to achieve the synthesis of super-aligned arrays, one has to control some of the parameters, such as partial pressure and temperature, to guarantee a much higher axial growth rate than AC deposition rate.

Since the AC deposition rate is very high at AP, we have to keep a very low mole ratio of acetylene to carrier gas, which can be argon, helium, or nitrogen. In case of an 1 in. tube furnace, the synthesis was carried out with $12 \mathrm{sccm}$ acetylene plus $425 \mathrm{sccm}$ argon gas at temper- atures ranging from 620 to $700^{\circ} \mathrm{C}$, which can give rise to super-aligned CNT arrays. However when we extended the synthesis to LP about 2 Torr, the low partial pressure can be automatically guaranteed since the total pressure is extremely low. In this case, we increased the mole ratio of acetylene to carrier gas to boost the axial growth rate. Uniform super-aligned arrays on a 4 in. wafer (Fig. 2A) can be achieved under the conditions of $500 \mathrm{sccm}$ acetylene plus $50 \mathrm{sccm}$ hydrogen at temperatures ranging from 680 to $720^{\circ} \mathrm{C}$. During the synthesis, the $4 \mathrm{in}$. substrate was placed horizontally in the middle of the tube furnaces. We also performed the growth by stacking a 2 in. substrate below the 4 in. one. Both give rise to a uniform super-aligned CNT array, indicating that the excellent uniformity can also be achieved along the perpendicular direction. This demonstrates that multi-wafer growth of superaligned CNT arrays in a big furnace is possible. In fact, this scale-up process can further be expanded to even larger scale in LP-CVD systems.

Practically, the following precautions turn out to be important in the synthesis of superaligned CNT arrays. 1) According to our results, the optimal temperature range for APCVD is from 620 to $700^{\circ} \mathrm{C}$, and for LP (2 Torr) CVD is from 680 to $720^{\circ} \mathrm{C}$. 2) The main requirement for the substrates is a polished flat surface. We have tried a silicon wafer, a thermal-oxidized silicon wafer, and a quartz plate as substrates, all can give rise to super-aligned arrays. Porous silicon, however, is not a very good substrate for super-aligned arrays. 3) Another key step is preparing densely packed catalyst particles with a narrow size distribution, which will result in CNTs with nearly the same diameter. Consequently the CNTs will have the same axial growth rate, which results in a perfect van der Waals bonding from top to end. To meet this requirement we use an electron-beam evaporator to prepare the iron film to be $3.5-5.5 \mathrm{~nm}$ thick at a very low deposition rate of about $0.1 \AA^{-1}$.

For the tensile testing of CNT yarns, we use an Instron 5848 microtester at a strain rate of $2 \% \mathrm{~min}^{-1}$ for a $3 \mathrm{~cm}$ gauge length. Note that the cross section of the yarn is usually irregular with some hollow cavity inside (Fig. 2I). Therefore, to get the tensile strength of the yarn, the first thing is to obtain the area of cross section exactly. According to the HRTEM image of as-grown CNTs, the outer diameter is about $15 \mathrm{~nm}$ and the inner diameter is about $5 \mathrm{~nm}$. We therefore take the density of our CNT as 8:9 of the density of graphite $\left(2.26 \mathrm{~g} \mathrm{~cm}^{-3}\right)$. By measuring the weight of a $2.6 \mathrm{~m}$ long ethanol treated yarn, which is $1.3 \mathrm{mg}$, we can obtain the effective area of cross section of $2.5 \times 10^{-6} \mathrm{~cm}^{2}$, which is used to convert the measured force to the stress factor.

Received: November 25, 2005

Final version: March 5, 2006

Published online: May 23, 2006

[1] a) S. Iijima, Nature 1991, 354, 56. b) Carbon Nanotubes: Preparation and Properties (Ed: T. W. Ebbesen), CRC, Boca Raton, FL 1997. c) P. J. F. Harris, Carbon Nanotubes and Related Structures: New Materials for the Twenty-first Century, Cambridge University Press, Cambridge, UK 1999. d) M. S. Dresselhaus, G. Dresselhaus, P. Avouris (Eds.), Carbon Nanotubes: Synthesis, Structure, Properties, and Applications, Springer, Heidelberg, Germany 2001

[2] a) M. Terrones, Annu. Rev. Mater. Res. 2003, 33, 419. b) R. H. Baughman, A. A. Zakhidov, W. A. de Heer, Science 2002, 297, 787.

[3] a) H. M. Cheng, F. Li, X. Sun, S. D. M. Brown, M. A. Pimenta, A. Marucci, G. Dresselhaus, M. S. Dresselhaus, Chem. Phys. Lett. 
1998, 289, 602. b) Z. W. Pan, S. S. Xie, B. H. Chang, C. Y. Wang, L. Lu, W. Liu, W. Y. Zhou, W. Z. Li, L. X. Qian, Nature 1998, 394, 631. c) H. W. Zhu, C. L. Xu, D. H. Wu, B. Q. Wei, R. Vajtai, P. M. Ajayan, Science 2002, 296, 884. c) S. Huang, X. Cai, J. Liu, J. Am. Chem. Soc. 2003, 125, 5636. d) L. X. Zheng, M. J. O'Connel, S. K. Doorn, X. Z. Liao, Y. H. Zhao, E. A. Akhadov, M. A. Hoffbauer, B. J. Roop, Q. X. Jia, R. C. Dye, D. E. Peterson, S. M. Huang, J. Liu, Y. T. Zhu, Nat. Mater. 2004, 3, 673.

[4] a) C. Liu, H. M. Cheng, H. T. Cong, F. Li, G. Su, B. L. Zhou, M. S. Dresselhaus, Adv. Mater. 2000, 12, 1190. b) B. Vigolo, A. Penicaud, C. Coulon, C. Sauder, R. Pailler, C. Journet, P. Bernier, P. Poulin, Science 2000, 290, 1331. c) S. Kumar, T. D. Dang, F. E. Arnold, A. R. Bhattacharyya, B. G. Min, X. Zhang, R. A. Vaia, C. Park, W. W. Adams, R. H. Hauge, R. E. Smalley, S. Ramesh, P. A. Willis, Macromolecules 2002, 35, 9039. d) A. B. Dalton, S. Collins, M. Munoz, J. M. Razal, V. H. Ebron, J. P. Ferraris, J. N. Coleman, B. G. Kim, R. H. Baughman, Nature 2003, 423, 703. e) Y. L. Li, I. A. Kinloch, A. H. Windle, Science 2004, 304, 276. f) L. M. Ericson, H. Fan, H. Peng, V. A. Davis, W. Zhou, J. Sulpizio, Y. Wang, R. Booker,
J. Vavro, C. Guthy, A. N. G. Parra-Vasquez, M. J. King, S. Ramesh, R. K. Saini, C. Kittrell, G. Lavin, H. Schmidt, W. W. Adams, W. E. Billups, M. Pasquali, W. F. Hwang, R. H. Hauge, J. E. Fischer, R. E. Smalley, Science 2004, 305, 1447.

[5] K. Jiang, Q. Li, S. Fan, Nature 2002, 419, 801.

[6] M. Zhang, K. R. Atkinson, R. H. Baughman, Science 2004, 306, 1358.

[7] M. Zhang, S. Fang, A. A. Zakhidov, S. B. Lee, A. E. Aliev, C. D. Williams, K. R. Atkinson, R. H. Baughman, Science 2005, 309, 1215.

[8] P. Liu, Y. Wei, K. L. Jiang, Q. Sun, X. B. Zhang, S. S. Fan, S. F. Zhang, C. G. Ning, J. K. Deng, Phys. Rev. B: Condens. Mat. Mater. Phys., in press.

[9] a) W. Z. Li, S. S. Xie, L. X. Qian, B. H. Chang, B. S. Zou, W. Y. Zhou, R. A. Zhao, G. Wang, Science 1996, 274, 1701. b) Z. F. Ren, Z. P. Huang, J. W. Xu, J. H. Wang, P. Bush, M. P. Siegal, P. N. Provencio, Science 1998, 282, 1105

[10] S. Fan, M. G. Chapline, N. R. Franklin, T. W. Tombler, A. M. Cassel, H. Dai, Science 1999, 283, 512. 